involving few controlled studies with selected patients. (see Progress in Pediatric Neurology II, 1994. pp 516-19, for other articles on diet and behavior).

\title{
ASPERGER SYNDROME AND AUTISM COMPARED
}

The validity and neuropsychological characterization of Asperger syndrome (AS) was investigated by comparison with Higher-Functioning Autism (HFA) (ie autism associated with overall normal intelligence) in 73 potential subjects recruited from a consecutive case series seen at the Developmental Disabilities Clinic, Child Study Center, Yale University School of Medicine, New Haven, CT. A full scale IQ $>70$ and a diagnosis of AS or autism according to ICD-10 research criteria were required for inclusion. The sample selected included 21 with AS and 19 with HFA. The AS group had a higher Verbal IQ and lower Performance IQ in comparison with the HFA group. There was a significant interaction between clinical diagnosis and IQ type. A high degree of concordance between AS and the condition of Nonverbal Learning Disabilities (NLD) was observed when overlap between psychiatric diagnosis (ie AS/HFA) and neuropsychological characterization (ie NLD assets and deficits) was examined. The NLD profile was an adequate neuropsychological model for individuals with AS but not for HFA. The AS and HFA groups differed significantly in 11 neuropsychological areas. (Klin A et al. Validity and neuropsychological characterization of Asperger syndrome: convergence with nonverbal learning disabilities syndrome. I Child Psychol Psychiat Oct 1995;36:1127-1140). (Reprints: Ami Klin, Child Study Center, Yale University School of Medicine, 230 South Frontage Road, New Haven CT 06520).

COMMENT. The neuropsychological profile obtained for children with Asperger syndrome (AS) coincided with that of the nonverbal learning disabilities syndrome (NLD) and differed from patients with higherfunctioning autism. The defining criteria for NLD included assets in auditory perception and memory, vocabulary, and spelling, and deficits in motor skills, visual-motor integration, visual-spatial perception, visual memory, verbal content, reading comprehension, arithmetic, and social and emotional competence. The authors suggest that intervention strategies for AS should differ from those for autism, directly addressing specific neuropsychological deficits and building on

neuropsychological assets, an approach found useful in individuals with nonverbal learning disabilities syndrome.

\section{BRAIN TUMORS}

\section{SIGNS OF BRAIN TUMORS IN INFANTS}

The presenting symptoms and signs of intracranial tumors diagnosed before the age of 2 years in 21 children treated at the University Hospital of Wales are reported from the Department of Child Health, Cardiff, UK. Nine of the tumors were supratentorial and 12 were infratentorial. The commonest tumor types were astrocytoma in 5 , ependymoma 4 , and medulloblastoma in 4 . The interval between onset of symptoms and diagnosis ranged from less than 1 day to 7 months. Nine had symptoms for at least 3 months before diagnosis. CT or MRI and biopsies were performed in all cases. The commonest presenting symptoms were vomiting in 9, and unsteadiness in 8 . The commonest presenting sign was enlarged head circumference in 16. Meningitis was 